Original Research Paper

\title{
Vehicle-Fixed-Frame Adaptive Controller and Intrinsic Nonlinear PID Controller for Attitude Stabilization of a Complex-Shaped Underwater Vehicle
}

\author{
${ }^{1}$ Chanaka Thushitha Bandara, ${ }^{2}$ Lakmali Nadeesha Kumari, ${ }^{3}$ Sanjeeva Maithripala and ${ }^{3}$ Asanga \\ Ratnaweera
}

${ }^{1}$ Department of Mechanical Engineering, University of Delaware, Newark, DE 19716, USA

${ }^{2}$ Department of Electrical and Electronic Engineering, University of Peradeniya, KY 20400, Sri Lanka

${ }^{3}$ Department of Mechanical Engineering, University of Peradeniya, KY 20400, Sri Lanka

\begin{abstract}
Article history
Received: 24-09-2020

Revised: 02-12-2020

Accepted: 02-12-2020

Corresponding Author:

Chanaka Thushitha Bandara,

Department of Mechanical

Engineering, University of

Delaware, Newark, DE 19716,

USA

E-mail: chanaka@udel.edu
\end{abstract}

\begin{abstract}
In this study we present attitude stabilization using a vehiclefixed-frame adaptive controller and an intrinsic nonlinear PID controller for a low-speed Autonomous Underwater Vehicle (AUV), of complex shape. Controlling an AUV poses a huge challenge because of the non-linearity, time variance and unpredictable external disturbance, as well as because its dynamics and hydrodynamic parameters are difficult to identify due to its geometry. First, a vehicle-fixed-frame adaptive controller is implemented to stabilize the attitudes given. The stability of this desired state-dependent, regressor, matrix-based controller is verified using Lyapunov's direct approach. Second, an intrinsic nonlinear PID controller is implemented based on the attitude error represented as rotation matrices, to stabilize the attitudes given. This controller requires only an approximate estimate of the inertia tensor of the device. Both the controllers adopt quaternions to indicate the attitude errors to avoid representation of the singularities that occur when the Euler angle description of the orientation is used. Finally, the results imply that the intrinsic nonlinear PID controller has more stability and shorter settling time than does the vehicle-fixed-frame adaptive controller.
\end{abstract}

Keywords: Autonomous Underwater Vehicle (AUV), Attitude Stabilization, Adaptive Control, PID Control, Quaternions

\section{Introduction}

Over the last two decades, Autonomous Underwater Vehicles (AUVs) have come in a wide variety of types and shapes and exerted a great influence on underwater applications. Areas inaccessible by even the Remote Operating Vehicles (ROVs) and Human Occupied Vehicles (HOVs) can be reached by the small-sized, complex-shaped AUVs because of their greater flexibility. These AUVs are also operable in highly hazardous environments and high-risk regions. Meanwhile, the complex-shaped AUVs have more numbers of Degrees of Freedom (DOF) than do the conventional torpedo-shaped ones, in terms of raising the maneuverability in complex underwater spaces.

As the AUV applications are increasing, the need for its autonomy is also escalating. Among the pivotal ways the AUV autonomy can be enhanced is to raise the dynamic working of its motion control system. Yamamoto (2001) demonstrated that a model-based control system is more effective when the AUV dynamics are known, at least to some degree. Furthermore, as Ferreira et al. (2012) observed an empirical model most frequently fails to illustrates the AUV dynamics over an extensive operating region. Therefore, to achieve an accurate hydrodynamic model of the complex-shaped AUVs it becomes important to design a controller. In order to model the hydrodynamics of these underwater vehicles, the scaled and full-scale experiments and computational approaches available at present, are the methods being used. However, scaled and full-scale experiments involve the use of costly devices like towing tanks, which makes them very expensive methods to employ (Berge and Fossen, 1997) and also, the computational approaches are required high computational 
time because of its complexity (Phillips et at., 2010). Building a suitable controller for the AUVs is a complicated process because of the complexity of the hydrodynamic parameters.

Several control systems are proposed to track the attitudes and trajectories to enable the AUV to behave as a highly refined machine for oceanic cruising and operation (Ismail et al., 2016; Xiang et al., 2017). The sliding mode controller is well known for its robustness, as all the relevant facts regarding the input nonlinearities and disturbances are unknown. In fact, Cui et al. (2016) suggested an adaptive, dynamic, anti-wind-up, sliding mode, attitude controller, on the basis of a simplified second-order dynamics and the use of a nonlinear disturbance observer and an auxiliary dynamic compensator were proposed for the situation of deadzone nonlinearity and rudder. To offset the ocean currents-induced unreliability in the hydrodynamics and unpredictable disturbance effects, a second-order sliding mode controller was developed by Joe et al. (2014). For tracking the desired trajectories, it was Eng et al. (2008) who addressed the horizontal tracking control for the AUVs on the basis of a nonlinear sliding mode incremental feedback model. Employing the Line Of Sight (LOS), Wang et al. (2012) initiated a feedback controller for the purpose of tracking the trajectories specified in the presence of ocean currents; In their work, Repoulias and Papadopoulos (2007) introduced a sliding mode controller, centered on the approach of the LOS as well as cross-track error.

However, as the chattering phenomenon is one of the major drawbacks of the conventional sliding mode controller, the adaptive concept can be utilized to minimize it (Zhang et al., 2014). In the presence of constant and ocean disturbance, Dong et al. (2015) in their study, suggested a state feedback-based, backstepping control algorithm. Further improvement of the controller was done to raise the steady-state performance, through an additional integral action, using the expression of the relationship between the heading angle and direction angle of the ocean current. Just as Smith et al. (2010) emphasized the desired trajectories were tracked by a wheeled robot which was controlled by utilizing a linearized fuzzy adaptive controller provided with backstepping feedback. With the help of the adaptive tracking controller, based on the Radial Basis Function Neural Network (RBF-NN) (Chwa, 2011), the trajectory tracking of the AUVs was accomplished. In fact, Bian et al. (2012) utilized the Dynamic Surface Control (DSC) and Minimal Learning Parameters (MLP), based on the robust adaptive neural network tracking control for underwater vehicles. In order to model the stability and robustness of the adaptive controller for a nonholonomic robot, Miao et al. (2013) adopted the learning method of the neural network. In their study, Bandara et al. (2019a) proposed the trajectory tracking and path planning algorithm based on an adaptive control law to facilitate the operation of a complexshaped low-speed Autonomous Underwater Vehicle (AUV) under challenging environmental conditions.

The PID controller is one of the most widely used controllers in underwater vehicle motion control. The VORTEX underwater vehicle was experimented upon using an improved nonlinear PID to subdue the nonlinearity and overcome the external interference, as addressed by Perrier and Canudas-De-Wit (1996). The results of this study were compared with the conventional PID controller. A PID depth controller based on the nonlinear model of an underwater vehicle was designed by Adhami-Mirhosseini et al. (2011). The 3-D path was suggested by Tian et al. (2011) following the controller of the underactuated AUV in the in the tack which followed the designs of the guidance function and PID controller. The fuzzy logic theory was adopted for improvement of the guidance function. In fact, Liu et al. (2005) applied a PID controller having the capacity to reject the shallow water wave disturbance to the ODIN underwater vehicle. Considering the effects of the ocean currents on the MKII underwater vehicle, a PID controller was implemented by Refsnes et al. (2007).

In the literature reports are available on techniques that are recommended for following the attitudes and trajectory tracking methods in the AUVs. However, in the course of designing suitable controllers, the main difficulties encountered include the uncertainty of the hydrodynamic parameters, such as the non-linear hydrodynamic effects, variations in the parameters and disturbances due to the ocean currents, as well as the nonlinear dynamics of the AUV. To maneuver the AUV in the desired manner, a controller that can overcome such problems is required.

In the present work, for the simulations the complexshaped AUV employed is displayed in Fig. 1, while the details of the configuration are given in Table 1.

Table 1: Characteristics of the complex-shaped AUV

\begin{tabular}{ll}
\hline Size & $(\mathrm{L}) 0.525,(\mathrm{~W}) 0.406,(\mathrm{H}) 0.395$ \\
\hline Weight in air & $16.00 \mathrm{~kg}$ \\
Propulsion & 4 horizontal and 2 vertical propellers \\
Degrees of freedom & Surge, Sway, Heave, Roll, Pitch, Yaw \\
Speed & $0-0.6 \mathrm{~m} / \mathrm{s}$ \\
\hline
\end{tabular}



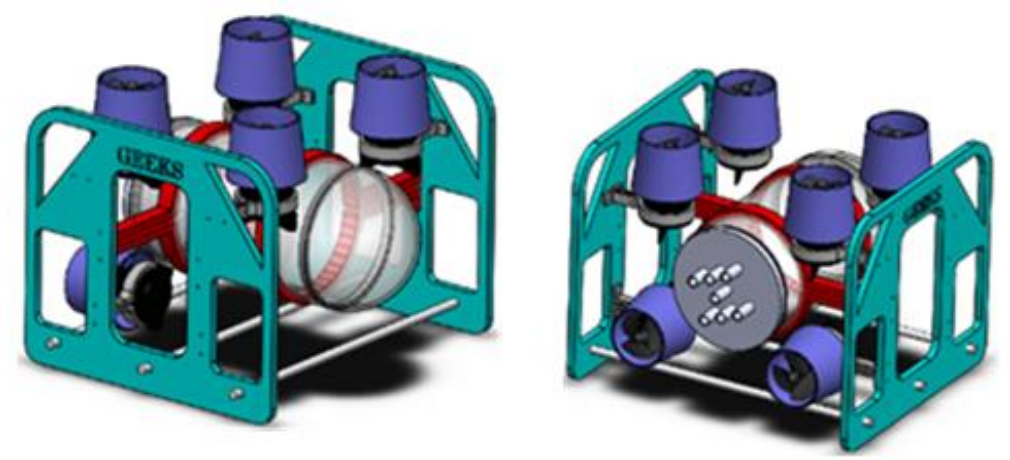

Fig. 1: 3D CAD Images of the AUV (GrabCAD, 2020)

This study presents a vehicle-fixed-frame adaptive controller and an intrinsic nonlinear PID controller for a complex-shaped AUV to stabilize the attitudes given. In Section AUV modeling, AUV modeling and standard notions for marine vehicles are introduced. To track the desired attitudes in the presence of hydrodynamic uncertainties, two Sections, namely Vehicle-fixed-frame adaptive controller and Intrinsic nonlinear PID controller focus on the development of a vehicle-fixed frame adaptive controller and an intrinsic nonlinear PID controller, respectively. Finally, in the Conclusion Section, the conclusions drawn are included, based on the performance comparison of both the controllers used, in Section Performance comparison of the vehicle-fixed-frame adaptive controller and Intrinsic nonlinear PID controller.

\section{AUV Modeling}

In this section the AUV kinematics and dynamics are clearly depicted, indicating the highly non-linear and coupled terms, which poses a challenge for the mathematical model. It was Fossen (2002) who, for convenience, introduced the North East Down (NEDframe) and Body fixed frame (B-frame) as the two coordinate frames for marine systems, as seen in Fig. 2.

It was Fossen (2002) who proposed the AUV dynamics based on the marine vehicle formulation; further, Fossen (1994) and the Society of Naval Architects and Marine Engineers (SNAME) (SNAME, 1950). In Table 2, the positions, angles, linear and angular velocities, force and moment are clearly evident. To ensure clarity, we have defined the position vector $(\eta)$, velocity vector $(v)$ and force vector $(\tau)$, as shown below:

$$
\begin{aligned}
& \eta=[x, y, z, \phi, \theta, \psi]^{\mathrm{T}} \\
& v=[u, v, w, p, q, r]^{\mathrm{T}} \\
& \tau=[X, Y, Z, K, M, N]^{\mathrm{T}}
\end{aligned}
$$

The AUV can be modeled as a rigid body because the relative distance between the particles remains fixed. The AUV dynamics as given by Euler's rigid body equation of motion, are defined as follows: (4) and (5) for the linear motion and rotational motion, respectively:

$$
\begin{aligned}
& M R_{I}^{B^{T}} \ddot{O}_{b}+M\left(v_{2} \times v_{2} \times \bar{X}+\dot{v}_{2} \times \bar{X}\right)=\tau_{1}+\Delta \tau_{1} \\
& I \dot{v}_{2}+v_{2} \times I v_{2}-M R_{I}^{B^{T}} \ddot{O}_{n} \times \bar{X}=\tau_{2}+\Delta r_{2}
\end{aligned}
$$

where, $M$ is the mass, $I$ is the moment of inertia defined in the B-frame, $\bar{X}$ is the center of mass defined in the B-Frame and $\Delta \tau 1$ and $\Delta \tau 2$ are the external disturbance force and moment defined in the B-frame, respectively. $R_{I}^{B} \in S O(3)$ is the rotation matrix with respect to the NED-frame:

The AUV kinematics is defined as follows:

$\dot{R}_{I}^{B}=R_{I}^{B} \widehat{v}_{2}$

The rotation matrix satisfies $R_{I}^{B^{T}} R_{I}^{B}=I_{3 \times 3}$ and angular velocity is $v_{2}$ defined by $\widehat{v}_{2}=R_{I}^{B^{T}} \dot{R}_{I}^{B}$, where $\widehat{v}_{2}$ is the skew symmetric version of $v_{2} \in \mathbb{R}^{3}$ The isomorphism between $\mathbb{R}^{3}$ and $s o(3)$, the space of $3 \times 3$ skew symmetric matrices given by ${ }^{-}: \mathbb{R}^{3} \rightarrow \operatorname{so}(3)$, is explicitly expressed as:

$\dot{v}_{2}=\left[\begin{array}{ccc}0 & -r & q \\ r & 0 & -p \\ -q & p & 0\end{array}\right] \epsilon \operatorname{so}(3)$

The assumption taken to model the AUV given in Fig. 1 is noted, as follows.

Assumption: The origin of the B-frame $\left(O_{b}\right)$ coincides with the center of the mass of the AUV; then $\bar{X}$ is equal to $\left[\begin{array}{lll}0 & 0 & 0\end{array}\right]^{T}$. 


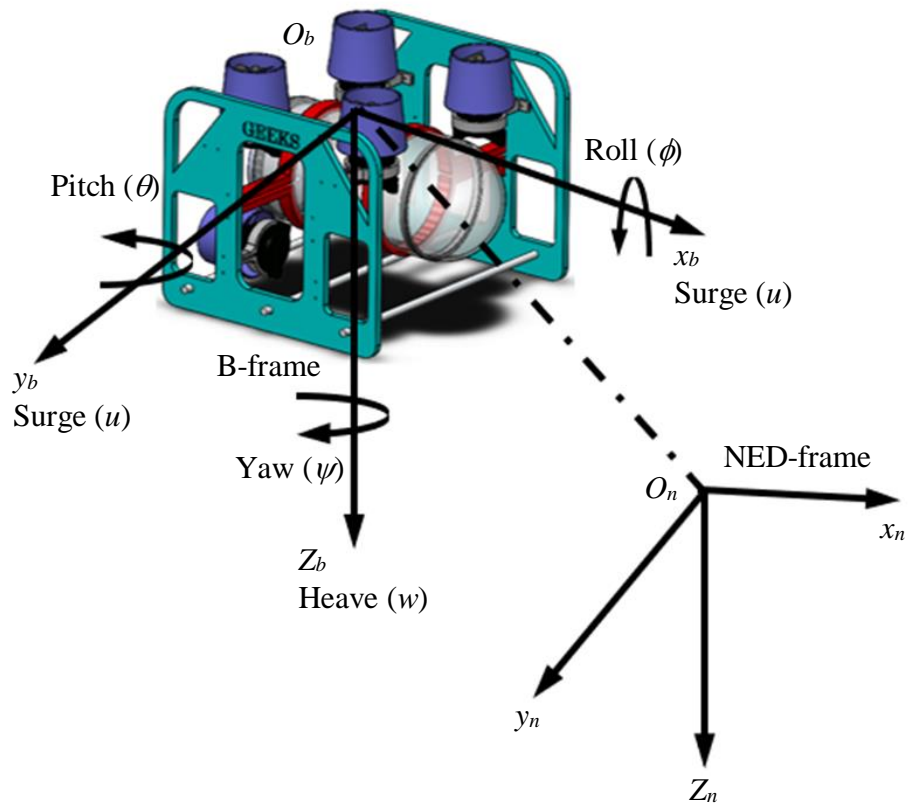

Fig. 2: NED frame and B-frame of underwater vehicles

Table 2: The SNAME notation for marine vessels

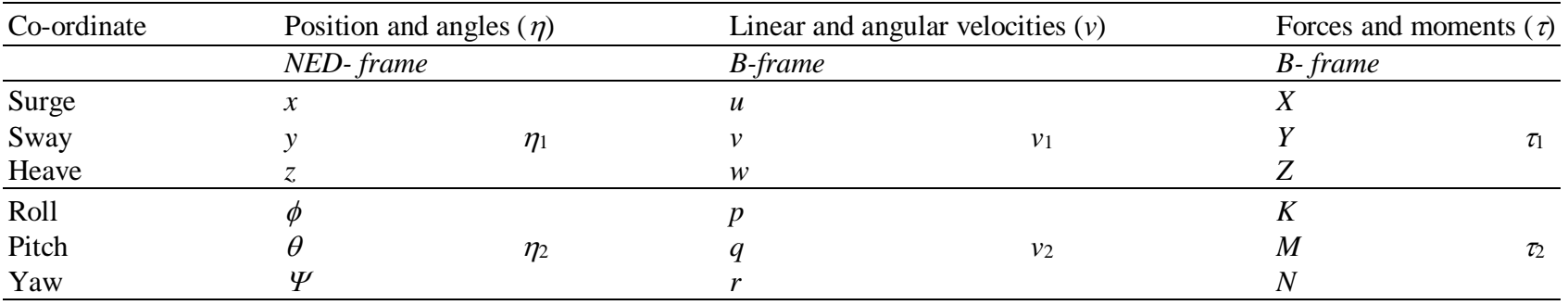

\section{Vehicle-Fixed-Frame Adaptive Controller}

From the data in (4) and (5), the dynamics of the AUV denoted reveal the uncertainty of the parameters and unmodeled dynamics. The design was created to compensate for these uncertainties; therefore, to achieve consistent AUV performance a vehicle-fixed-frame adaptive controller is suggested to estimate and control the uncertain parameters. Using the by the adaption mechanism, adjustment of the parameters in the control law is done. The design of the adaptive controller proposed is capable of forcing the AUV to maintain, in the presence of parameter uncertainty, the attitudes desired. To accomplish this, the regression matrix is defined.

The vehicle-fixed variables, are considered, as given:

$$
\begin{aligned}
& \tilde{\vartheta}=f\left(R_{I}^{B^{T}} \eta_{2 d}-R_{I}^{B^{T}} \eta_{2}\right) \\
& \tilde{v}_{2}=v_{2 d}-v_{2}
\end{aligned}
$$

where the quaternion-based attitude error is given by $\tilde{\vartheta}$, $\eta_{2 d}$ is the desired attitude and $v_{2 d}$ is the desired angular velocity of the AUV in the body-fixed frame.

The error vector is given by:

$S=\tilde{v}_{2}+\Lambda \tilde{\vartheta}$

where, $\Lambda$ is a positive definite matrix.

The vehicle regressor matrix is:

$\Phi=\left(v_{2}, \dot{v}_{2}, v_{2 d}, \dot{v}_{2 d}\right)$

The adaptive control law can be defined as expressed below:

$\tau_{2}=\Phi\left(v_{2}, \dot{v}_{2}, v_{2 d}, \dot{v}_{2 d}\right) \widehat{\Theta}+K_{D} S$

Such that:

$I \dot{v}_{2}+v_{2} \times I v_{2}=\Phi\left(v_{2}, \dot{v}_{2}, v_{2 d}, \dot{v}_{2 d}\right) \widehat{\Theta}+K_{D} S$ 
where, $K_{D}$ is the $(3 \times 3)$ positive definite matrix.

The parameter estimate $\widehat{\Theta}$ is updated by:

$$
\hat{\dot{\Theta}}=\Gamma \Phi^{T} S
$$

where, $\Gamma$ is a suitable positive definite matrix of appropriate dimension and is selected in such a manner that the tuning law provides the convergent characteristics.

In the equation, the adaptive law proposed can drive the AUV in the desired direction, thus guaranteeing the AUV stability. In light of the discussion mentioned above, the structure of the proposed control law is depicted in the block diagram given in Fig. 3.

Stability of the Vehicle-Fixed-Frame Adaptive Controller

The following Lyapunov candidate function is used to examine the stability of the system:

$V(t)=\frac{1}{2}\left[S^{T} I S+\tilde{\Theta}^{\mathrm{T}} \Gamma^{-1} \tilde{\Theta}\right]=V_{1}(t)+V_{2}(t)$,

$\tilde{\Theta}=\tilde{\Theta}-\Theta, \forall S \neq 0, \tilde{\Theta} \neq 0$

where, $V_{1}(t)=\frac{1}{2} S^{T} I S$ and $V_{2}(t)=\frac{1}{2} \tilde{\Theta}^{\mathrm{T}} \Gamma^{-1} \tilde{\Theta}$.

The selected Lyapunov candidate function satisfies the conditions given in (16):

$V(t): R^{n} \rightarrow R$ such that $V(t) \geq 0$ if and only if

$t=0($ positive definite $) \dot{V}(t)=\frac{d V(t)}{d t} \leq 0$,

if and only if $t=0$ (negative definite)

In reality, the system is asymptotically stable in the sense of Lyapunov because $V(t)$ conforms to the conditions stated above. The time derivative of (15) is expressed as given below:

$\dot{V}(t)=\dot{V}_{1}(t)+\dot{V}_{2}(t)$

Being $\dot{V}_{1}(t)$ and $\dot{V}_{2}(t)$ as follows:

$\dot{V}_{1}(t)=S^{\mathrm{T}} I \dot{S}+\frac{1}{2} S^{\mathrm{T}} \dot{I}$

$\dot{V}_{2}(t)=\hat{\dot{\Theta}}^{\mathrm{T}} \Gamma^{-1} \tilde{\Theta}$

where, $\dot{\tilde{\Theta}}=\widehat{\dot{\Theta}}+0$, as $\Theta$ is a constant definite vector

By the substitution of (10) in (18), according to Bandara et al. (2019b):
$\dot{V}_{1}(t)=S^{\mathrm{T}} I\left(\dot{v}_{2 d}-\dot{v}_{2}\right)+\frac{1}{2} S^{\mathrm{T}} \dot{I} S$

where, $\dot{S}=\dot{v}_{2 d}-\dot{v}_{2}$.

Substituting the value of $I \dot{v}_{2}$ from (5) in (20) and simplifying $\dot{V}_{1}(t)$ :

$\dot{V}_{1}(t)=S^{\mathrm{T}}\left(I \dot{v}_{2 d}+v_{2} \times I v_{2}-\tau_{2}\right)+\frac{1}{2} S^{\mathrm{T}} \dot{I} S$

$\dot{V}_{1}(t)=S^{\mathrm{T}}\left(I \dot{v}_{2 d}+\left(-s+v_{2 d}\right) \times I\left(-s+v_{2 d}\right)-\tau_{2}\right)+\frac{1}{2} S^{\mathrm{T}} \dot{I} S$

$\dot{V}_{1}(t)=S^{\mathrm{T}}\left(I \dot{v}_{2 d}+v_{2 d} \times I v_{2 d}-\tau_{2}+s \times I S\right.$

$\left.+I v_{2 d} \times s+s I \times v_{2 d}\right)+\frac{1}{2} S^{\mathrm{T}} \dot{I S}$

$\dot{V}_{1}(t)=S^{\mathrm{T}}\left(I \dot{v}_{2 d}+v_{2 d} \times I v_{2 d}-\tau_{2}\right)+\frac{1}{2} S^{\mathrm{T}}$

$\left(\left(\dot{I}+2 I_{3 \times 3}\left(s \times I s+I v_{2 d} \times s+s I \times v_{2 d}\right)\right) S\right)$

$\dot{I}+2 I_{3 \times 3}\left(s \times I s+I v_{2 d} \times s+s I \times v_{2 d}\right)$ is, for AUVs, a skewsymmetric matrix. By substituting the system dynamics shown in (5) into the equation mentioned:

$\dot{V}_{1}(t)=S^{T}\left(\Phi \Theta-\tau_{2}\right)$

Therefore, Equation (17) becomes:

$\dot{V}(t)=S^{\mathrm{T}}\left(\Phi \Theta-\tau_{2}\right)+\hat{\stackrel{\Theta}{\mathrm{\Theta}}}^{\mathrm{T}} \Gamma^{-1} \tilde{\Theta}$

Substituting control input $\left(\tau_{2}\right)$ into (26):

$\dot{V}(t)=S^{\mathrm{T}}\left(\Phi \widehat{\Theta}-\Phi-K_{D} S\right)+\widehat{\dot{\Theta}}^{\mathrm{T}} \Gamma^{-1} \tilde{\Theta}$

$\dot{V}(t)=S^{\mathrm{T}}\left(-\Phi \dot{\Theta}-K_{D} S\right)+\dot{\Theta}^{\mathrm{T}} \Gamma^{-1} \tilde{\Theta}$

$\dot{V}(t)=-S^{\mathrm{T}} \Phi \dot{\Theta}-S^{\mathrm{T}} K_{D} S+\hat{\dot{\Theta}}^{\mathrm{T}} \Gamma^{-1} \widehat{\Theta}$

Substituting $\hat{\Theta}$ from (14) in (29)

$\dot{V}(t)=-S^{\mathrm{T}} \Phi \dot{\Theta}-S^{\mathrm{T}} K_{D} S+\left(\Gamma \Phi^{\mathrm{T}} S\right)^{\mathrm{T}} \Gamma^{-1} \tilde{\Theta}$

$\dot{V}(t)=-S^{\mathrm{T}} K_{D} S \leq 0$

Equation 31, with the use of a stable controller, complies with the Lyapunov criterion of the stability of the AUV dynamics. Therefore, the adaptive control law proposed in (12) provides a closed system which is stable. 


\section{Intrinsic Nonlinear PID Controller}

This intrinsic nonlinear PID controller is implemented to recompense the uncertainty of the parameters and unmodeled dynamics, included in the AUV dynamics, denoted in (4) and (5). Implementing the controller necessitates knowing only the inertia tensor of the device. However, a broad estimation of the parameters of the system will enable the identification of a suitable gain set, using simulation.

The rotational motion dynamics given by (5) can be rearranged as (32) to simplify the control algorithm:

$\dot{v}_{2}=I^{-1}\left(I v_{2} \times v_{2}\right)+T^{u}+\Delta T$

where, $\tau_{2}=I T^{u}$ represents the control moments generated by the thrust of the rotors. The $\Delta \tau_{2}=I \Delta T$ term includes both the uncertainty of the parameters and external disturbances:

According to Somasiri et al. (2015), $R_{I d}^{B}(t) \epsilon S O(3)$, the following explicit controller described, has the capacity to locally, exponentially and almost globally stabilize an adequately smooth reference trajectory:

$$
\begin{aligned}
& E=R_{I d}^{B}(t)^{T} R_{I}^{B}(t) \\
& \hat{\eta}_{E}=R_{I}^{B}(t) R_{I d}^{B}(t)^{T}-R_{I d}^{B}(t) R_{I}^{B}(t)^{T} \\
& \hat{\Omega}_{e}=E^{T} \dot{E} \\
& \dot{\Omega}_{i}=-\frac{1}{2}\left(v_{2} \times \Omega_{i}-I^{-1}\left(I \Omega_{i} \times v_{2}+I v_{2} \times \Omega_{i}\right)\right)+\eta_{E} \\
& T^{u}=-k_{p} \eta_{E}-k_{d} \Omega_{e}-k_{i} \Omega_{i}
\end{aligned}
$$

where, $k_{p}, k_{d}$ and $k_{i}$ are the positive gains, the tracking error is shown in Equation 33; the gradient of the error function $f(E)=\operatorname{Trace}\left(I_{3 \times 3}-E\right)$ is revealed in Equation 34; the intrinsic integrator is depicted in Equation 36 and the nonlinear intrinsic PID control action is represented in Equation 37:

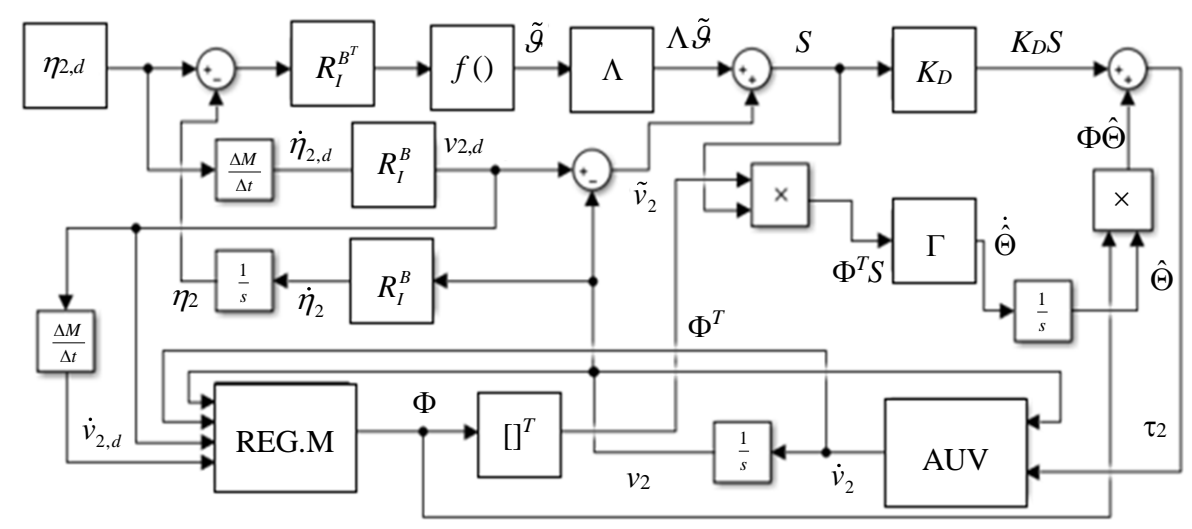

Fig. 3: MATLAB SIMULINK layout of the vehicle-fixed-frame adaptive controller

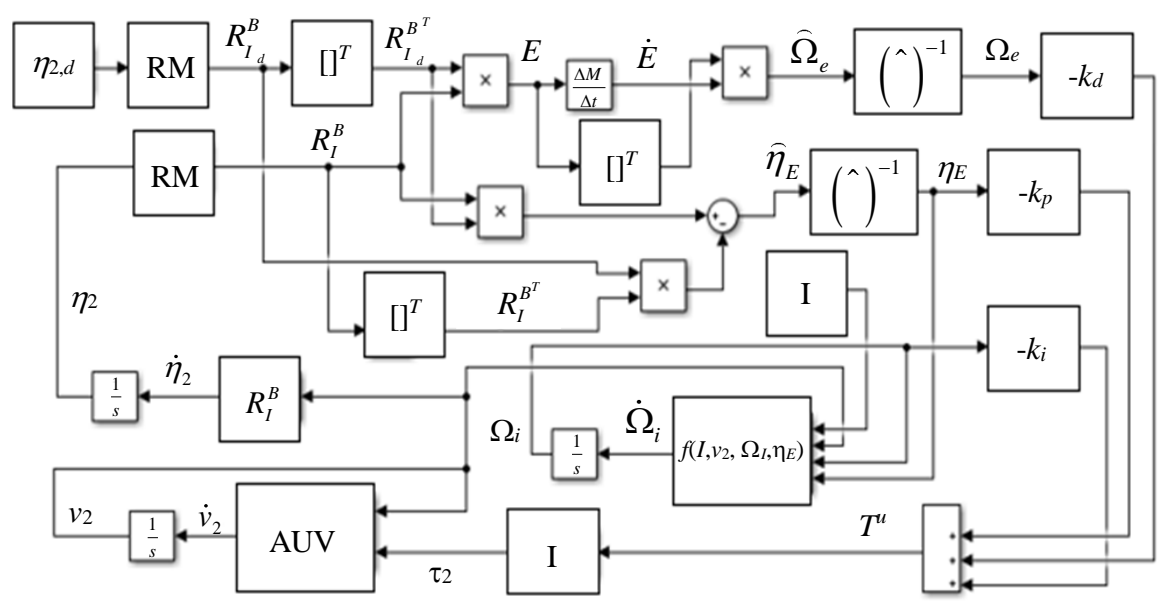

Fig. 4: MATLAB SIMULINK layout of the intrinsic nonlinear PID controller 
The structure of the control law proposed, as discussed above, is depicted in Fig. 4.

\section{Performance Comparison of the Vehicle- Fixed-Frame Adaptive Controller and Intrinsic Nonlinear PID Controller}

In this section, to identify the stability of the proposed controllers, simulations for the AUV given in Fig. 1 were conducted, in the presence of external disturbances.

For this simulation, four DOF are considered: Surge, roll, pitch and yaw. The force required to maneuver the AUV in the $x_{b}$ direction and maintain a given velocity is manually set to the desired value. The added mass acting in the $x_{b}$ direction when the AUV operates at low speed can be neglected compared to its mass (Bandara et al., $2019 b$ ). The details of the AUV configuration and damping torques, which can be identified as the external disturbances $\left(\Delta \tau_{2}\right)$ applied to it, can be listed as (38) and Table 3 (Bandara et al., 2019a):

$$
I=\left[\begin{array}{ccc}
0.2568 & 0 & 0 \\
0 & 0.3841 & 0 \\
0 & 0 & 0.3716
\end{array}\right] \mathrm{kgm}^{2}
$$

The desired attitudes and heading velocity are defined in Table 4. The roll DOF ( $\phi$ ) behaves like a selfstabilizer, so that the desired roll DOF $\left(\phi_{d}\right)$ is equal to zero throughout the given time period.

Then, the initial position, attitudes and velocity of the AUV are $(x, y, z)=(0,0,0),(\phi, \theta, \Psi)=(0,0,0)$ and $(u, v, w)=$ $(0,0,0)$, respectively.
The Control Law Parameters of the Vehicle-Fixedframe Adaptive Controller

$$
\tau_{2}=\Phi\left(v_{2}, \dot{v}_{2}, v_{2 d}, \dot{v}_{2 d}\right) \widehat{\Theta}+K_{D} S \text { is the control input of }
$$
this controller, as defined in (12). Its gain matrices are selected to be, as Table 5,

In the event of using the regression matrix $(\Phi)$, the AUV model shown in (5) can be given in terms of linear parameters. To implement it in real-time, it must be understood that the regression matrix, which is statedependent, is needed to calculate, in each control cycle (Bandara et al., 2019a). The regression matrix defined in (11) can be given as shown below:

$$
\Phi=\left[\begin{array}{cccccc}
\dot{p}_{d} & \left|p_{d}\right| p_{d} & 0 & 0 & 0 & 0 \\
0 & 0 & \dot{q}_{d} & \left|q_{d}\right| q_{d} & 0 & 0 \\
0 & 0 & 0 & 0 & \dot{r}_{d} & \left|r_{d}\right| r_{d}
\end{array}\right]
$$

\section{The Control Law Parameters of the Intrinsic Nonlinear PID Controller}

As defined in (37), $T^{u}=-k_{p} \eta_{E^{-}} k_{d} \Omega_{e^{-}} k_{i} \Omega_{i}$ is the control input of this controller. The inertia tensor $(I)$ according to (38) is used in implementing the nonlinear integrator expressed in (36). The nonlinear PID controller gains selected were $k_{p}=\left[\begin{array}{lll}60 & 60 & 45\end{array}\right] k_{d}=\left[\begin{array}{ll}35 & 35\end{array}\right.$ 25] and $k_{i}=\left[\begin{array}{lll}10 & 10 & 5\end{array}\right]$.

The number of simulations is performed for the desired attitudes given in Table 4 to check the performance of the proposed controllers.

Fig. 5a and $5 \mathrm{~b}$ show the comparison between the actual attitudes and desired attitudes with each controller and elaborate that the intrinsic nonlinear PID controller shows the low settling time and greater stability than the vehicle-fixed-frame adaptive controller.

Table 3: Estimated damping torque with angular velocity

\begin{tabular}{ll}
\hline Direction & Damping torque $\left(\Delta \tau_{2}\right) /(\mathrm{Nm})$ \\
\hline Roll & $\Delta \tau_{2 r}=0.29 p^{2}+0.291|\mathrm{p}|$ \\
Pitch & $\Delta \tau_{2 p}=0.531 q^{2}+0.373|\mathrm{q}|$ \\
Yaw & $\Delta \tau_{2 r}=0.345 r^{2}+0.125|r|$ \\
\hline
\end{tabular}

Table 4: The desired attitudes in NED-frame and heading velocity of the AUV in B-frame used for the simulation.

\begin{tabular}{lllcr}
\hline Time $(t) /(\mathrm{s})$ & $\begin{array}{l}\text { Linear velocity in } \\
x_{b} \text { direction }(u) /(\mathrm{m} / \mathrm{s})\end{array}$ & $\begin{array}{l}\text { Desired roll } \\
\text { angle }\left(\phi_{d}\right) /\left(^{\circ}\right)\end{array}$ & $\begin{array}{l}\text { Desired pitch } \\
\text { angle }\left(\theta_{d}\right) /\left(^{\circ}\right)\end{array}$ & $\begin{array}{r}\text { Desired yaw } \\
\text { angle }\left(\Psi_{d}\right) /\left(^{\circ}\right)\end{array}$ \\
\hline $0-5$ & 0.4 & 0 & 0 & 0 \\
$5-10$ & 0.4 & 0 & -20 & 20 \\
$10-15$ & 0.4 & 0 & -40 & 30 \\
$15-20$ & 0.4 & 0 & -10 & 10 \\
$20-25$ & 0.4 & 0 & 10 & -5 \\
$25-30$ & 0.4 & 0 & 45 & -20 \\
$30-35$ & 0.4 & 0 & 60 & -35 \\
$35-40$ & 0.4 & 0 & 50 & -25 \\
$40-45$ & 0.4 & 0 & 35 & -15 \\
$45-50$ & 0.4 & 0 & 20 & 0 \\
\hline
\end{tabular}


Table 5: Gain matrices

\begin{tabular}{|c|c|}
\hline Gain & Matrix \\
\hline$K_{D}$ & $\operatorname{diag}\left(\left[\begin{array}{lll}20 & 10 & 10\end{array}\right)\right.$ \\
\hline$\Lambda$ & $\operatorname{diag}\left(\left[\begin{array}{lll}25 & 20 & 20\end{array}\right]\right)$ \\
\hline$\Gamma$ & $\operatorname{diag}\left(\left[\begin{array}{llllll}20 & 20 & 15 & 15 & 15 & 15\end{array}\right]\right)$ \\
\hline
\end{tabular}

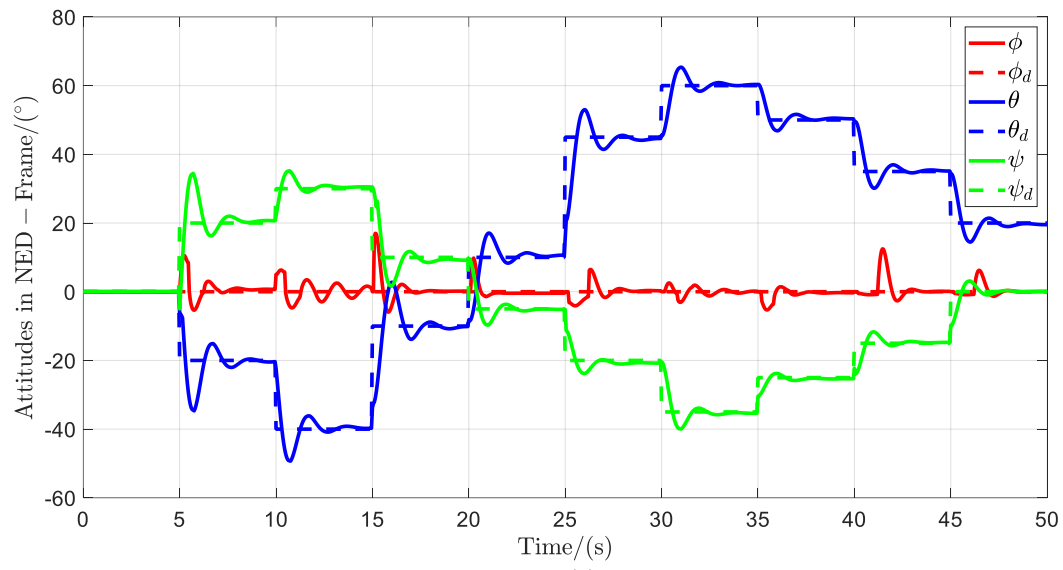

(a)

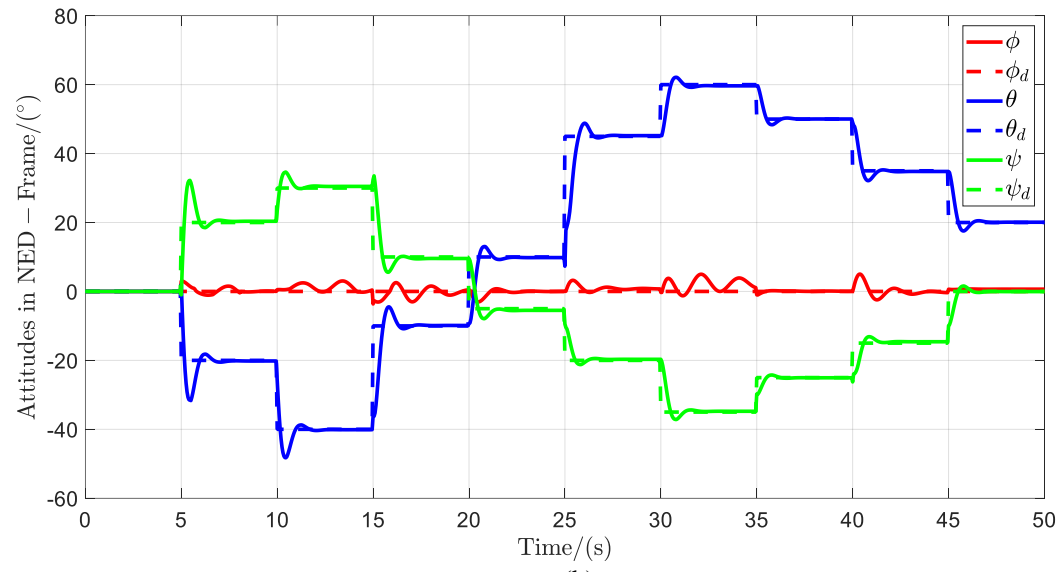

(b)

Fig. 5: (a) Actual attitude and desired attitude of the AUV with the vehicle-fixed-frame adaptive controller (b) Actual attitude and desired attitude of the AUV with the intrinsic nonlinear PID controller.

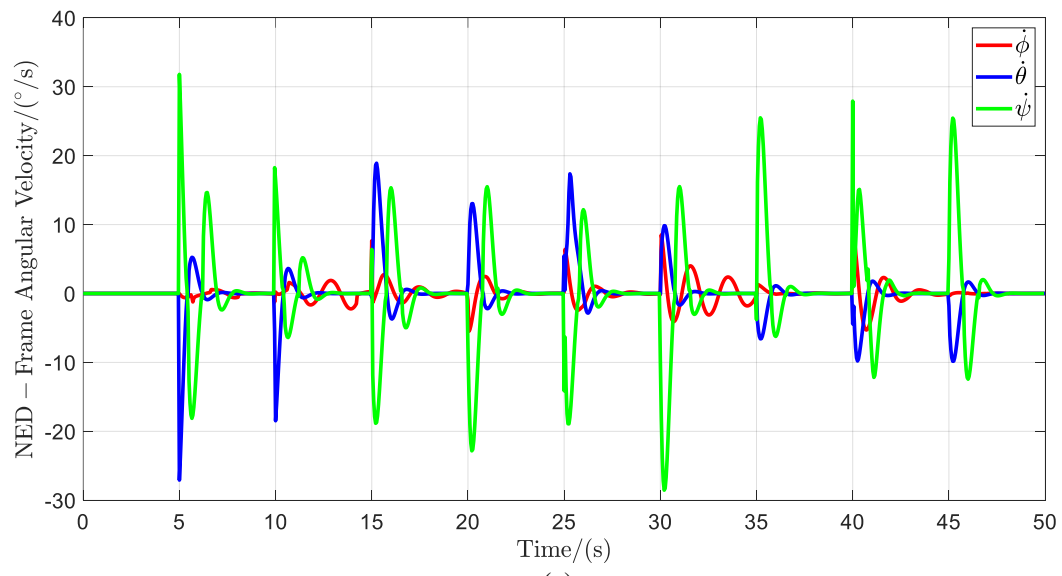

(a) 


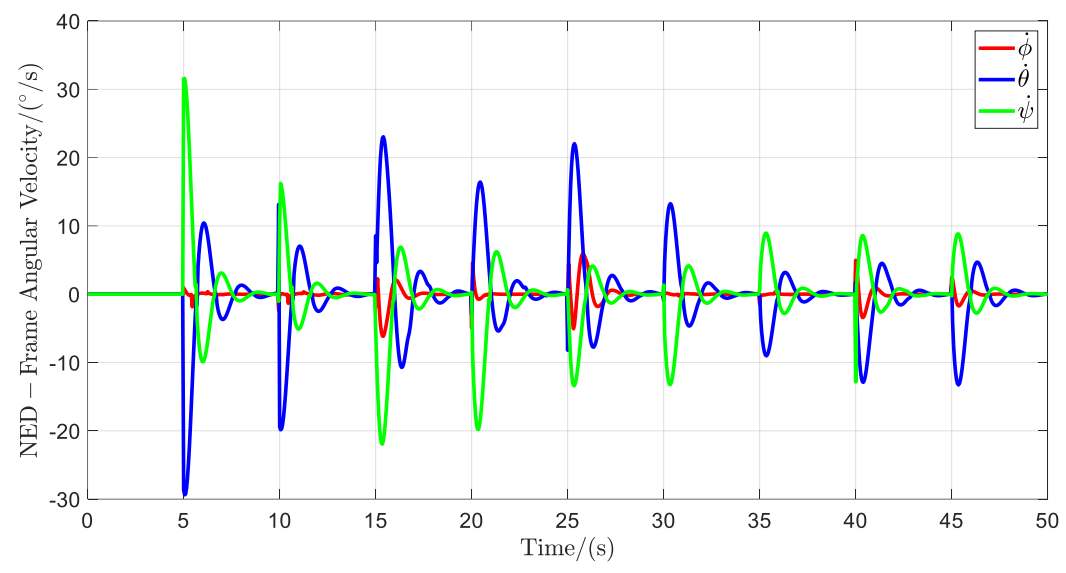

(b)

Fig. 6: (a) NED-frame angular velocity of the AUV with the vehicle-fixed-frame adaptive controller (6b) NED-frame angular velocity of the AUV with the intrinsic nonlinear PID controller

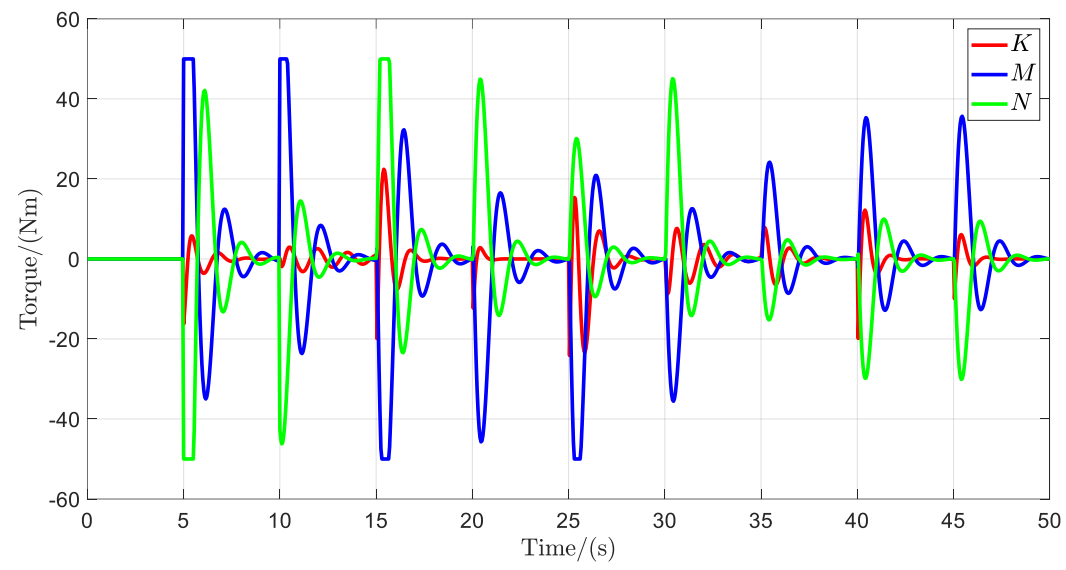

(a)

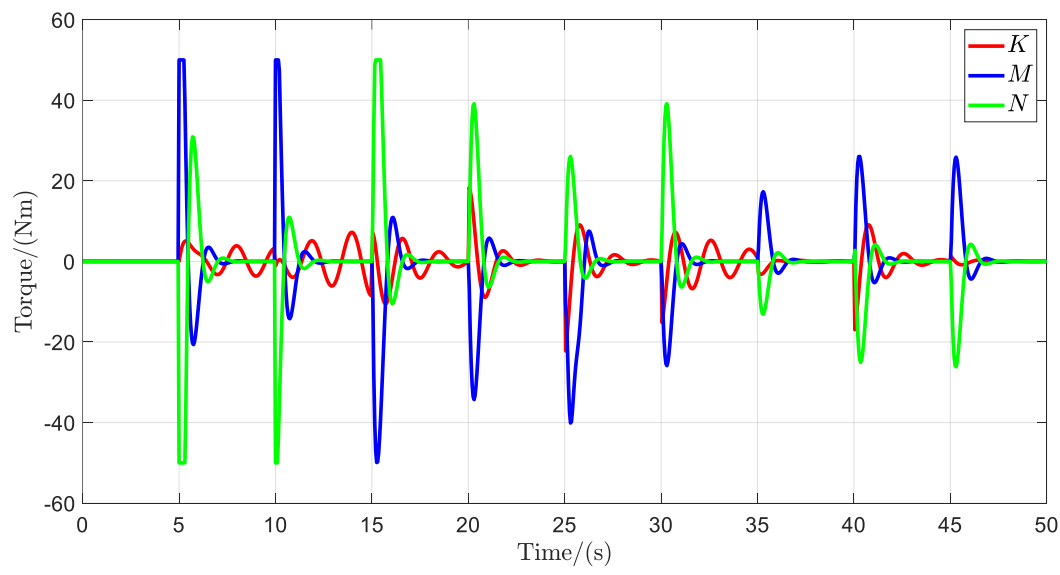

(b)

Fig. 7: (a) Control moments of the AUV with the vehicle-fixed-frame adaptive controller (b) Control moments of the AUV with the intrinsic nonlinear PID controller 
In Fig. $6 a$ and $6 b$, it is evident that the NED-frame angular velocities of the AUV with the intrinsic nonlinear PID controller asymptotically converge to zero faster than does the vehicle-fixed-frame adaptive controller.

In Fig. $7 a$ and $7 b$, at the beginning of each desired attitude, the control moments have certain definite values indicating that the AUV reaches to the required angular acceleration. After that, the control moments asymptotically converge to zero, as the angular accelerations decrease to zero, proposing that each controller accurately and smoothly commands the AUV to follow the desired attitudes. The control outputs are constrained between -50 $\mathrm{Nm}$ and $50 \mathrm{Nm}$ given that all the thrusters have limitations on proving the maximum propulsion moments.

\section{Conclusion}

This study considers the problems of attitude stabilization for a complex-shaped AUV, in the presence of external disturbances. Moreover, Euler's rigid body equations of motion used to model the AUV can easily handle the linear and rotational motions, separately. Also, the quaternion representation of the attitudes guarantees avoidance of the singularities. The simulation results demonstrate the comparison between the attitude stabilization of the vehicle-fixed-frame adaptive controller and the intrinsic nonlinear PID controller to be effective and robust to external disturbances. Lyapunov's direct approach is used to confirm the stability of the vehicle-fixed-frame adaptive controller, which is rather more complicated than the intrinsic nonlinear PID controller, which only requires a rough estimation of the inertia tensor. From these results it is evident that the intrinsic nonlinear PID controller has faster convergence and greater stabilization than does the vehicle-fixed-frame adaptive controller. Also, to the best of our knowledge, this intrinsic nonlinear PID controller provides the best simulation results reported for an attitude stabilization controller, implemented for a complex-shaped underwater vehicle.

\section{Acknowledgement}

This section should be state the contributions made by each author in the preparation, development and publication of this manuscript.

\section{Author's Contributions}

Chanaka Thushitha Bandara:

- Conceptualization

- Methodology

- Investigation

- Software

- Visualization
- Writing-original draft preparation

- Writing-review and editing

Lakmali Nadeesha Kumari:

- Methodology

- Formal analysis

- Software

- Writing-original draft preparation

- Writing-review and editing

Sanjeeva Maithripala:

- Supervision

Asanga Ratnaweera:

- Supervision

\section{Ethics}

This article is original and contains unpublished material. Authors declare that are no ethical issues and no conflict of interest may arise after the publication of this manuscript.

\section{References}

Adhami-Mirhosseini, A., Aguiar, A. P., \& Yazdanpanah, M. J. (2011, December). Seabed tracking of an autonomous underwater vehicle with nonlinear output regulation. In 2011 50th IEEE Conference on Decision and Control and European Control Conference (pp. 3928-3933). IEEE.

Bandara, G. A. C. T., Ratnaweera, D. A. A. C., \& Maithripala, D. H. S. (2019a). Modeling and Control Approach for a Complex-Shaped Underwater Vehicle. American Journal of Mechanical Engineering, 7(4), 158-171.

Bandara, G. A. C. T., Ratnaweera, D. A. A. C., \& Maithripala, D. H. S. (2019b, December). Hydrodynamic Modelling and Adaptive Controlling for a Complex-Shaped Underwater Vehicle. In 2019 14th Conference on Industrial and Information Systems (ICIIS) (pp. 372-377). IEEE.

Berge, S. P., \& Fossen, T. I. (1997). Robust control allocation of overactuated ships; experiments with a model ship. IFAC Proceedings Volumes, 30(22), 193-198.

Bian, X., Zhou, J., Yan, Z., \& Jia, H. (2012, March). Adaptive neural network control system of path following for AUVs. In 2012 Proceedings of Ieee Southeastcon (pp. 1-5). IEEE.

Chwa, D. (2011). Fuzzy adaptive tracking control of wheeled mobile robots with state-dependent kinematic and dynamic disturbances. IEEE transactions on Fuzzy Systems, 20(3), 587-593. 
Cui, R., Zhang, X., \& Cui, D. (2016). Adaptive slidingmode attitude control for autonomous underwater vehicles with input nonlinearities. Ocean Engineering, 123, 45-54.

Dong, Z., Wan, L., Li, Y., Liu, T., Zhuang, J., \& Zhang, G. (2015). Point stabilization for an underactuated AUV in the presence of ocean currents. International Journal of Advanced Robotic Systems, 12(7), 100.

Eng, Y. H., Lau, M. W. S., Low, E., Seet, G., \& Chin, C. S. (2008). Estimation of the Hydrodynamics Coefficients of an ROV using Free Decay Pendulum Motion. Engineering Letters, 16(3), 326-331.

Ferreira, B. M., Matos, A. C., \& Cruz, N. A. (2012, April). Modeling and control of trimares auv. In 12th International Conference on Autonomous Robot Systems and Competitions (pp. 57-62).

Fossen, T. (1994). Guidance and Control of Ocean Vehicles. John Willey \& Sons. Inc., New York.

Fossen, T. I. (2002). Marine Control Systems-Guidance. Navigation and Control of Ships, Rigs and Underwater Vehicles. Marine Cybernetics, Trondheim, Norway, Org. Number NO 985195005 MVA, www. marinecybernetics. com, ISBN: 8292356002.

GrabCAD. (2020). AUV design. GrabCAD Community Library. https://grabcad.com/library/rov-54

Ismail, Z. H., Mokhar, M. B., Putranti, V. W., \& Dunnigan, M. W. (2016). A robust dynamic regionbased control scheme for an autonomous underwater vehicle. Ocean Engineering, 111, 155-165.

Joe, H., Kim, M., \& Yu, S. C. (2014). Second-order sliding-mode controller for autonomous underwater vehicle in the presence of unknown disturbances. Nonlinear Dynamics, 78(1), 183-196.

Liu, S., Wang, D., Poh, E. K., \& Chia, C. S. (2005, September). Nonlinear output feedback controller design for tracking control of ODIN in wave disturbance condition. In Proceedings of OCEANS 2005 MTS/IEEE (pp. 1803-1810). IEEE.

Miao, B., Li, T., \& Luo, W. (2013). A DSC and MLP based robust adaptive $\mathrm{NN}$ tracking control for underwater vehicle. Neurocomputing, 111, 184-189.

Perrier, M., \& Canudas-De-Wit, C. (1996). Experimental comparison of PID vs. PID plus nonlinear controller for subsea robots. In Underwater Robots (pp. 121138). Springer, Boston, MA.

Phillips, A. B., Turnock, S. R., \& Furlong, M. (2010). The use of computational fluid dynamics to aid costeffective hydrodynamic design of autonomous underwater vehicles. Proceedings of the Institution of Mechanical Engineers, Part M: Journal of Engineering for the Maritime Environment, 224(4), 239-254.
Refsnes, J. E., Sørensen, A. J., \& Pettersen, K. Y. (2007). Output feedback control of slender body underwater vehicles with current estimation. International Journal of Control, 80(7), 1136-1150.

Repoulias, F., \& Papadopoulos, E. (2007). Planar trajectory planning and tracking control design for underactuated AUVs. Ocean engineering, 34(1112), 1650-1667.

Smith, R. N., Chao, Y., Li, P. P., Caron, D. A., Jones, B. H., \& Sukhatme, G. S. (2010). Planning and implementing trajectories for autonomous underwater vehicles to track evolving ocean processes based on predictions from a regional ocean model. The International Journal of Robotics Research, 29(12), 1475-1497.

SNAME, T. (1950). Nomenclature for treating the motion of a submerged body through a fluid. The Society of Naval Architects and Marine Engineers, Technical and Research Bulletin No, 1-5.

Somasiri, J. A. A. S., Gamagedara, K. G. B., Maithripala, D. H. S., \& Berg, J. M. (2015, December). Implementation of an almost globally stable intrinsic nonlinear PID controller for attitude stabilization of a quadrotor. In 2015 IEEE 10th International Conference on Industrial and Information Systems (ICIIS) (pp. 425-428). IEEE.

Tian, Y., Zhang, A., \& Li, W. (2011, July). 3D pathfollowing of underactuated autonomous underwater vehicles. In Proceedings of the 30th Chinese control conference (pp. 3456-3461). IEEE.

Wang, L., Zhang, L. J., Jia, H. M., \& Wang, H. B. (2012, June). Horizontal tracking control for AUV based on nonlinear sliding mode. In 2012 IEEE International Conference on Information and Automation (pp. 460-463). IEEE.

Xiang, X., Yu, C., \& Zhang, Q. (2017). Robust fuzzy 3D path following for autonomous underwater vehicle subject to uncertainties. Computers \& Operations Research, 84, 165-177.

Yamamoto, I. (2001). Robust and non-linear control of marine system. International Journal of Robust and Nonlinear Control: IFAC-Affiliated Journal, 11(13), 1285-1341.

Zhang, X., Liu, X., \& Zhu, Q. (2014). Adaptive chatter free sliding mode control for a class of uncertain chaotic systems. Applied Mathematics and Computation, 232, 431-435. 